\section{Parametrization of Pedestrian Injuries and its Utilisation in Proving Traffic Accidents Course Using Injury Signatures and Contact Signatures}

\author{
Mandelík J. ${ }^{*}$, Bobrov N. ${ }^{2}$, Nevolná Z. ${ }^{3}$
}

\begin{abstract}
Background: The paper points out the present limited possibility of using the verbal description of injuries for the needs of experts from the field of road transportation as relevant criminalistics traces, as well as the options of the FORTIS system that creates a new area for a deeper interdisciplinary approach in the field of expert evidence. Further a description of how to create injury signatures and contact signatures and the possibilities of their evaluation and mutual comparison based on the proven individual attributes are described
\end{abstract}

Objective: To evaluate pedestrian injuries by the new proper FORTIS system and to show FORTIS valuability in the assessment of mechanical violence and mechanism of injuries.

Material and Methods: Cases of traffic injuries including photodocumentation, graphic schemes, medical files and autopsy protocols processed by the new FORTIS forensic system.

Results: A collision between a pedestrian and a vehicle represents a matrix of physical violence having an effect on the pedestrian's body and a matrix of the pedestrian's body's response to this violence. The analysis of individual cases shows the valuability of the FORTIS system.

Conclusion: It is apparent that for the needs of traffic accidents analysts the FORTIS system has more options for being used in forensic medicine, as it covers not only a field of evaluation of traffic injuries but also all kinds of injuries and accidents (rail accidents, air accidents, violent crimes, etc.).

\section{Keywords}

Pedestrian, parametrization of injuries, collision analysis, forensic medicine, accident case analysis

\section{Introduction}

$\mathrm{T}$ he aim of this article is to get acquainted with the results of researching the possibilities of forensic parametrization and localization of pedestrian injuries resulting from a vehicle-pedestrian collision and with a consequent possibility to use the obtained information in solving and assessing an accident course by forensic engineers performing a technical analysis. The stated examples will be based on actual collision calculations and on a model of predicted pedestrian injuries due to a schematic explanation of the procedures used [1].

At present, an accident analyst, forensic engineer, has at solving the
${ }^{1}$ The University of Security Management in Kosice, ul. Kukučínova 17 04001 Kosice, Slovak

Republic

${ }^{2}$ Department of Foren-

sic Medicine, Faculty of

Medicine of University

Hospital of P. J. Šafárik

and University Hospital

of L. Pasteur in Košice,

Trieda SNP 1, 04011

Kosice, Slovak Republic

${ }^{3}$ Hospital of Horná

Orava with Health

Care Center in Trstená,

Mieru 549/16, 02801

Trstená, Slovak Republic 
accident case, considerably limited possibilities of using the information about manner and extent of injury of a pedestrian during accident case. It results from the fact that most often, it is only a verbal description of injuries elaborated by a physician; although, such a form of information only partially helps to disseminate necessary knowledge of the vehicle, pedestrian system. However, it is obvious that type, extent and localization of injuries of a pedestrian are some pieces of significant information about conditions and course of collision between a vehicle and a pedestrian. In the event that the information fails to correspondent with outcomes of technical analysis or simulation of collision, it is possible to consider it incomplete, or even in some cases incorrect, or in extreme cases to be technically unacceptable.

At solving the given issue, it is necessary:

- on the side of a medical examiners: to use knowledge in the field of forensic medicine in a form usable for analysis of collision, i.e. FORTIS system

- on the side of an analysts of accident case: to use computer technique for analysis (PC Crash or Virtual Crash)

which results in qualitative and comprehensive outcome of overall expert evidence.

\section{Injuries of Traffic Accident Par- ticipant as Criminalistic Traces}

An injury of a traffic accident participant (a pedestrian, crewmember, cyclist, motorcyclist) can be viewed and assessed from various angles.

\section{Legal Assessment}

It is based on the legal qualification of causes and consequences of injuries, on the investigation and demonstration of a degree of guilt on the part of participants as well as a degree of compensation of the affected persons in applying the respective legal provisions and procedures.

2. Medical Assessment

It is based on the diagnostics and subsequent treatment of incurred injuries and on the principle of reducing health consequences as well as on the quantification of injury extent for the needs of compensation of the affected persons.

3. Forensic Assessment

a. It approaches the injury evaluation in light of assessment of its severity for subsequent legal procedures within the investigation of a deed.

b. The evaluation of injuries as traces for the needs of interdisciplinary evidencing of a traffic accident course in cooperation with traffic accident analysts, in which the injury evaluation is approached in terms of assessing a degree of violence having an effect on the body of the affected person, causing the injuries, as well as a detailed localization of parts which were violated (FORTIS system) [2, 3].

The forensic assessment can be divided to:

- assessing the extent of injuries of people who did not survive the accident

- assessing the extent of injuries of people who survived the accident

From the investigational point of view, the outputs of an examination of an injured person should be in line with the needs of a forensic evaluation: i.e. photo documentation, description and a complete medical documentation.

Since the accident course is a unique and unrepeatable set of phenomena influenced by a variety of effects, out of which many are absolutely individual for a specific traffic accident, we may predict that in order to clarify, it is essential to use all known circumstances and facts, or that the performed traffic accident analysis and its subsequent assessment must fully correspond to all known facts and circumstances, whereas the extent and amount of the objectively known facts are largely influenced by the quality of the accident site inspection.

The traces on the bodies of victims and injured persons represent, as a whole, an integral part of the accident, and they carry the basic information about its course - especially in a collision phase, and as such, they must be 
a part of the accident analysis resources during the expert evidence. As the evaluation of the above- mentioned traces is carried out by forensic examiners, the question is to objectivize them by means of their localization and parametrization for further investigation needs and for the needs of traffic accident analysts. It is not a rare situation that the traffic accident analysts provide the documentation for forensic examiners in order to assess the possibility of occurrence or non-occurrence of the detected injuries.

Based on the above, we may consider pedestrian's injuries trace that are usable for the needs of evidencing of the accident course.
For that purpose, FORTIS system was developed, which enables us to make use of a forensic assessment of pedestrian injuries for an accident analysis as equivalent to other traces on a vehicle, road or other objects. FORTIS system itself then represents a sophisticated forensic assessment, which is usable for the purposes of a consequent technical analysis, at least as a verification element.

Nowadays, we can divide the classification, identification as well as assessment of injuries as traces by forensic examiners in relation to the engineering procedures of accident analysts into four development stages [4] (Table 1):

Table 1

\section{FORENSIC ENGINEERING - TRAFFIC AC- CIDENT ANALYSIS}

\section{1. stage}

A typical examination usually performed in a descriptive way using minimum or no exact methods, relying primarily on "experience"of an expert.

The first stage of the traffic accident analysis can be characterized as an analysis with minimal or no calculation, which is based on the expert's experience. The calculations and conclusions are only subjective and often incorrect.
RELATION

\section{1. stage}

A typical examination usually performed in a descriptive way using minimum or no exact methods, relying primarily on "experience" of an expert.

Possible incorrect conclusions of forensic examiners are associated mainly with Possible incorrect conclusions of forensic examiners are associated mainly with their technical ignorance of the actual accident course. A probability of the evidence beyond $a$ "belief"is non-existent and relies mainly on the trust in the knowledge of forensic examiners and the correctness of their judgement.

\section{2. stage}

A comparison with the known knowledge in the field connected with a simple modelling of the injured person's body's movement, perhaps even a general consultation with a technical expert without a detailed clarification of individual aspects of the occurrence of injuries.

A probability of incorrect conclusion has been partly removed, but a proof performed this way is still mostly based on the "experience and belief"of the expert and relies mainly on the trust in the knowledge of forensic examiners and in the correctness of their forensic judgement. 


\section{3. stage}

This stage makes use of the latest science and technology and applies two-dimensional and three-dimensional computer simulation programs for a traffic accident analysis. In these simulation programs various databases are used, in which technical parameters of vehicles and their tyres can be set. One of the advantages of the simulation programs is an ability to perform calculations for specific geometric road shapes with a choice of adhesion factors for each surface respectively and a choice to select vehicle chassis suspension stiffness and tyre characteristics. When using this stage of a traffic accident technical analysis an emphasis is placed on high-quality hardware and software equipment of computers, including high-quality input parameters, whereas a video-recording of a pedestrian/ vehicle movement is one of calculation outputs.

A probability of incorrect conclusions is low, but the elimination of alternatives is not sufficiently restricted - especially in terms of a position and movement of a pedestrian.

\section{4. stage}

This stage assumes a calculation in the simulation program is performed using detailed information about the injuries of participants, i.e. their localization and characteristics of the affecting violence and it assumes a subsequent consultation with a forensic examiner before a final assessment of an accident course and the accident itself.

A probability of incorrect conclusions is - with a proper use of the information and their evaluation - minimal, virtually removed, and the aforesaid procedure ensures that the performed proof has all features of exactness, completeness and controllability in relation to all detected traces including the traces on the injured person's body..

From the presented comparison of the method development, we may conclude that the completeness of evidence, with the current state of knowledge and with the currently applied methods, can be achieved mainly through the interdisciplinary evidence using the latest knowledge, procedures and methods of forensic engineering and forensic medicine.

We should emphasize that in order to determine an implementation stage of a specific interdisciplinary evidence, a lower development stage is decisive, i.e. the use of a higher stage in one sector in combination with a lower stage in another sector does not lead to an evidence

\section{3. stage}

A comparison with the known knowledge in the field connected with a cooperation with a technical expert to such an extent that the course of the movement of the injured body calculated in the simulation program is visually compared (a video recording of a collision calculation).

A probability of incorrect conclusions has been significantly removed. However, a proof performed this way is still based on the"experience "of the expert and on the way a technical expert calculates the collision, not using any exact knowledge of injuries, i.e. a type, localization or how they were caused.

\section{4. stage}

A detailed description of the detected injuries and their localization using the documented traces (measurements, photo documentation) and definition of the findings into a standardized form (e.g. Fortis) applicable for a technical expert as a description of the traces that must be considered in a collision calcula-

2 tion and their compliance with the forensic findings documented, a following consultation of a forensic examiner and a technical expert and an evaluation of the results of the calculation presented by a videorecording and an overview of contact parameters.

A probability of incorrect conclusion has been virtually removed and the aforesaid procedure ensures that the performed proof has all features of exactness, completeness and controllability in relation to all detected traces.

result at the level of the higher stage.

Parameterization of Injuries in Crashes Involving Pedestrian Injuries - FORTIS Aystem (Forensic Traumatology Injury Scale)

At present, the medical forensic analysis of traffic injuries is not always standardized, especially due to non-existence of an established, well-defined sequence for standardization of injury parameters. The proposed procedure for the assessment of traffic injuries, including the key element of injury parameter standardization according to internationally 
accepted AIS/ISS scale (Abbreviated Injury Scale/Injury Severity Score) is as follows:

\section{Material and Methods}

- Investigated circumstances, accompanying documentation, autopsy reports, image documentation and results of supplementary investigation evidence from traffic accidents involving pedestrian fatalities

- Complete autopsy report in accordance with the requirements of International Disease Classification (10th revision)

- Assessment of injury parameters using proprietary modified FORTIS system, comparison of FORTIS values with AIS/ ISS values.

\section{The Modified System FORTIS}

The result is a modified FORTIS, the use of which enables a more comprehensive definition of the extent of the injuries, thus enabling its application in the assessment of traffic accidents. FORTIS system uses a proprietary modified point value system, which besides determining the basic injury to health (ZPZ), proposes a classification of accompanying complications in two groups: Kol primary post-injury complications - such as traumatic shock, haemorrhagic shock, cardiac tamponade, hemothorax, pneumothorax) and secondary complications $\mathrm{Ko} 2$ - such as inflammatory changes, edemas of non-traumatic origin, thromboses, conditions arising from surgeries, etc. Such a division provides an opportunity to indirectly assess quality of healthcare provision in case of a surviving injured.

During the verification of modified FORTIS system itself, 12 fatal traffic accidents were subjected to assessment by forensic pathologists, evaluating each case as a single event, and subsequently each relevant item in the autopsy report was assessed in a similar way. The results acquired through above analysis are compared to standard assessment of injury parameters - AIS/ISS in order to demonstrate new possibilities of FORTIS in the quantification of injury parameters, especially in nonfatal traffic trauma involving pedestrians, to develop documentation for health insurance organizations $[3,5,6]$.

Presentation of FORTIS system and signature theory to be utilised when analysing collisions between vehicles and pedestrians as a complex accident action

Subsequently, to compare the methods, a point evaluation of 12 assessed pedestrian fatalities is introduced using standard AIS/ISS method, and the modified FORTIS system can be seen in Table 2.

It is apparent that the possibilities of FORTIS injury classification system are wider, the system is more flexible and relevantly differentiates the injuries resulting from the accident.

\section{Results}

\section{A. Results - case No.4}

Investigated circumstances: involving motor vehicle VW Passat, whereas the vehicle with its right side collided with underage pedestrian who entered its trajectory from the right side, causing him head injury to which he succumbed shortly after the accident. Figure 1

Immediate cause of death: Rupture of the connection between medulla oblongata and cerebral pons and contusion and laceration of brain and intracranial haemorrhage resulting from fragmented fractures of cranial calvaria and base. Figure 2

\section{B. Results - case No.11}

Investigated circumstances: involving a collision between passenger vehicle and pedestrian. The injured pedestrian was subsequently hospitalized at the traumatology department with bilateral fracture of pelvis (treated by conservative - Kirschner's extension of left leg). On 12th day of hospitalization, F.K. died. 
Table 2: Scoring of road accidents of pedestrians, using classical methodology AIS/ISS and respective modified FORTIS system.

\begin{tabular}{cccccccccc}
$\begin{array}{c}\text { Case } \\
\text { No. }\end{array}$ & Name, Age & M/W & Car & $\begin{array}{c}\text { Period of } \\
\text { survival } \\
\text { (hours) }\end{array}$ & ISS & $\begin{array}{c}\text { FORTIS } \\
\text { total }\end{array}$ & $\begin{array}{c}\text { FORTIS } \\
\text { ZPZ }\end{array}$ & $\begin{array}{c}\text { FORTIS } \\
\text { K01 }\end{array}$ & $\begin{array}{c}\text { FORTIS } \\
\text { K02 }\end{array}$ \\
\hline 1 & P. U.,41 years & M & Car & 0 & 66 & 44,1 & 22,4 & 11,7 & 13 \\
\hline 2 & M. J.,38 years & M & Car & 0 & 75 & 82,8 & 47,7 & 16,1 & 19 \\
\hline 3 & A. P.,67 years & W & Car & 0 & 75 & 78,2 & 53,6 & 17,6 & 7 \\
\hline 4 & E. P.,9 years & M & Car & 0 & 75 & 57,1 & 41,1 & 9 & 7 \\
\hline 5 & P. S.,49 years & M & Car & 0 & 75 & 66,3 & 65,3 & 1,0 & 0 \\
\hline 6 & A. C.,80 years & W & Car & 0.8 & 57 & 83,2 & 66,2 & 12 & 5 \\
\hline 7 & J. N.,58 years & M & Car & 2 & 75 & 97,4 & 77,4 & 7,3 & 12,7 \\
\hline 8 & J. B.,57 years & M & Car & 24 & 75 & 99,2 & 60,8 & 20,7 & 17,7 \\
\hline 9 & I. M.,47 years & M & Car & 72 & 75 & 102,7 & 46,9 & 16,1 & 37,7 \\
\hline 10 & S. Ch.,57 years & M & lorry & 239 & 75 & 59 & 19,1 & 14,5 & 25,4 \\
\hline 11 & F. K.,51 years & M & Car & 288 & 75 & 27 & 9,3 & 7,7 & 10 \\
\hline 12 & M. P.,39 years & M & Car & 291 & 75 & 60,8 & 24,2 & 8,5 & 28,1
\end{tabular}

Illustrative examples of scoring of forensic examination of fatal injuries with a different ratio ZPZ, Ko1 and Ko2

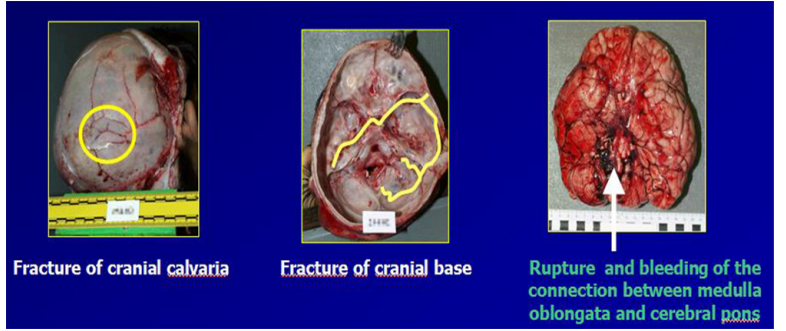

Figure 1: Documentation of finding - illustration to case no. 4

Figure 3

Immediate cause of death: Bilateral focal catarrhal-purulent broncho-pneumonia. Figure 4

\section{Evaluation and Benefits of the Mod- ified FORTIS system}

The results of the above-mentioned cases indicate that in case of pedestrian death at the site of accident, the point values yielded by FORTIS are relatively greater for items ZPZ and Kol, in case of surviving victim, the values of FORTIS Ko2 are progressively increasing, because there are more secondary complications, while the values of FORTIS Ko2 can be viewed also from the viewpoint

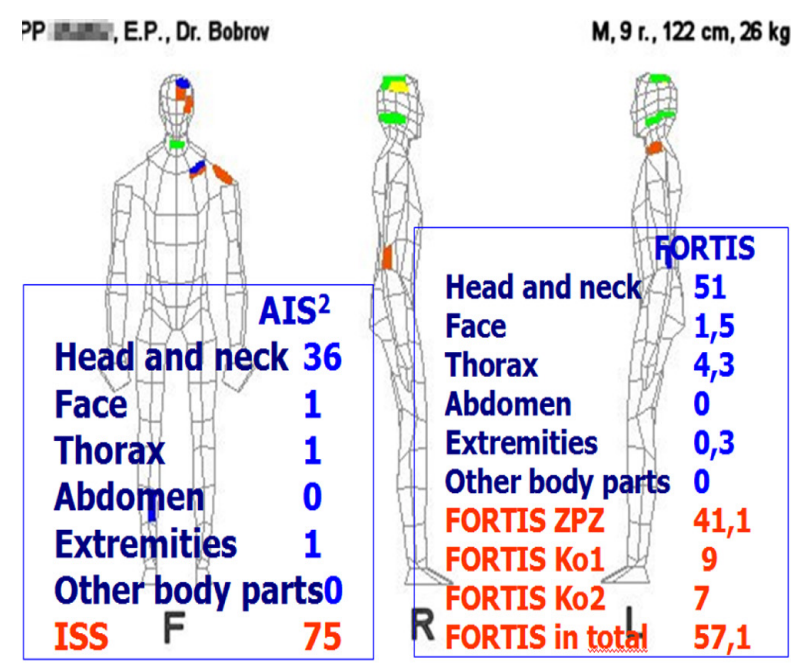

Figure 2: Evaluation of parameters of injuries in case no. 4 via custom modified FORTIS system and comparison of FORTIS values with AIS/ISS values

of the provision of healthcare, with the point value proportion of the basic injury to health, primary and secondary complications, is specific for each type of accident event.

The research conducted so far, outcomes of which are presented herein, as well as the 


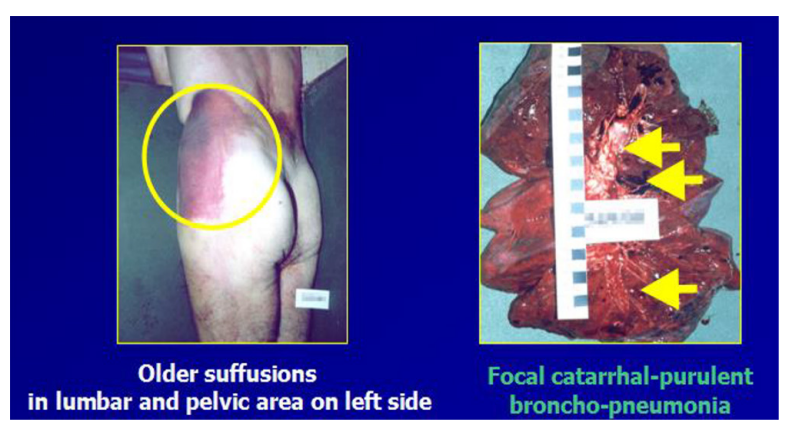

Figure 3: Documentation of the finding - illustration to case no. 11

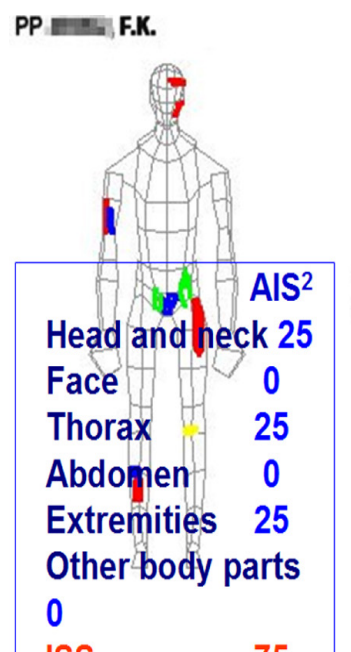

ISS

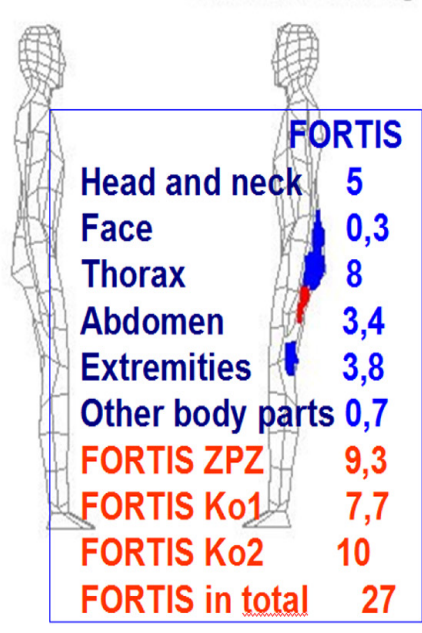

Figure 4: Evaluation of parameters of injuries in case no. 11 via custom modified FORTIS system and comparison of FORTIS values with AIS/ISS values

case studies of the already performed analyses enable us to state that the technique of standardization of pedestrian injury parameters in case of traffic accidents (quantification of injuries) requires the application of the proprietary modified scoring system FORTIS, that enables (subject to good quality of input parameters) to calculate the degree of basic injury to health, primary post-injury complications and secondary complications, including painful treatment procedures and poor quality of healthcare and in case of fatal injuries, it enables to determine the immediate cause of death.
It is also possible to use this standardized injury score to more accurately determine the degree of fault of a person responsible for the traffic accident from other circumstances, such as irresponsible attitude on the part of the victim or insufficient or inadequate treatment of the resulting injuries.

Traditional methods of injury parameter standardization (AIS/ISS) have proven to be insufficient with respect to the assessment of the extent of injuries, since - especially in case of higher energy acting upon the body the score does not change. On the other hand, FORTIS score reflects the extent of the injuries more accurately, thus making this quantification of injuries a basis for application using a computer calculation program (e.g. PC Crash) using which, it is possible to perform not only a simulation of accident sequence but also verification of its accuracy using FORTIS at pedestrians in connection with the localization of contact points on a pedestrian's body, as it is clear that FORTIS reflects the severity of injuries occurring during traffic accidents depending on the mechanical energy that caused the injuries during the impact [3, $7]$.

The modified system of injury scoring differs from the existing systems in the following aspects:

\section{FORTIS vS. AIS/ISS}

- Scoring of injuries and their complications using modified tables Injury Score in Forensic Medicine

- Special scoring of the basic injury to health (ZPZ)

- The complications are divided in two categories:

1) primary post-injury complications (Kol)

2) secondary complications (Ko2)

- The score values include decimal positions

- Maximum score of FORTIS is not limited

- Using the FORTIS methodology the point value of an injury is localized on the place of the injury causing contact on the surface of an injured person's body. 
It is clear that the above-mentioned differences between FORTIS and the scale of AIA/ ISS system significantly increase FORTIS utility, accuracy resulting in certain parameters of the injury expressed in the form of a number, enabling the generation of characteristic signatures with respect to the parameters of the collision between the vehicle and the pedestrian, or in general to the mechanism of injuries. An expert in the field of road transport would thus clearly understand which injuries are directly related to the accident (ZPZ) and which injuries are a result of the subsequent response of the pedestrian's body (Ko1), health condition, treatment procedures, etc. (Ko2), while it should be emphasized that FORTIS score is dimensionless and determined on the basis of a certain arbitrary scale.

At present, a total of 39 tables are available for scoring of injuries in individual parts of the body, with respect to critical health consequences, in respect of the applied level of forces. This data is gradually being validated and supplemented by additional data, and the method is being continuously improved $[2,3]$.

\section{PC FORTIS Program Designed for Pa-} rametrization and Localization of Injuries

A software application PC FORTIS was developed for the parametrization and visualization of injuries on the surface of the body. When a doctor uses this application during the first and during subsequent treatments, the quality of the data on pedestrian and his injuries should be greatly improved for the needs of traffic accident analysts. A 3D static strength prediction program - 3DSSPP (University of Michigan, USA, freeware) was used as auxiliary graphics software $[3,7]$.

An expert in the field of road transport should consider it a benefit to use or similar program which graphically presents the injuries, as it greatly improves the information on the contact localization and parameters of individual injuries.
When colour coding is used to distinguish between $\mathrm{ZPZ}, \mathrm{KOo} 1$ and $\mathrm{Ko} 2$, it can be assumed that such information will be significantly more valuable for the expert, whereas the tables with scores are located outside the dummy with identified localization of contacts on the body surface and colour coding of severity of individual injuries. It can be assumed that in case of primary medical intervention (traumatology in survivors), the doctor should have available a PC software showing the injuries identified by score value in colours corresponding to the assigned score.

PC FORTIS program, which in addition to the data on a patient (age, height, weight, clothes, verbally described diagnosis, etc.), enables a direct localization of a contact location on the body surface resulting in an injury and scoring of individual injuries using the provided classification tables and instructions for their use. Figures 5 and 6

The output presents a depiction of the localization of individual's injury contacts, overall point value as well as a local point value and its distribution on the body of a pedestrian patient. Figure 7

A detailed score - the result of FORTIS clas-

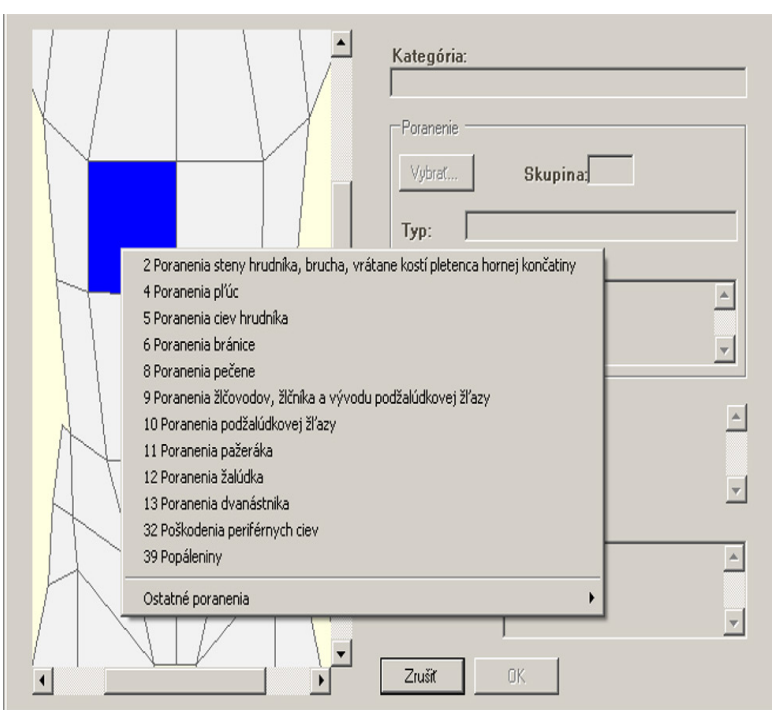

Figure 5: Illustration of the use of classification tables in the PC Fortis program 


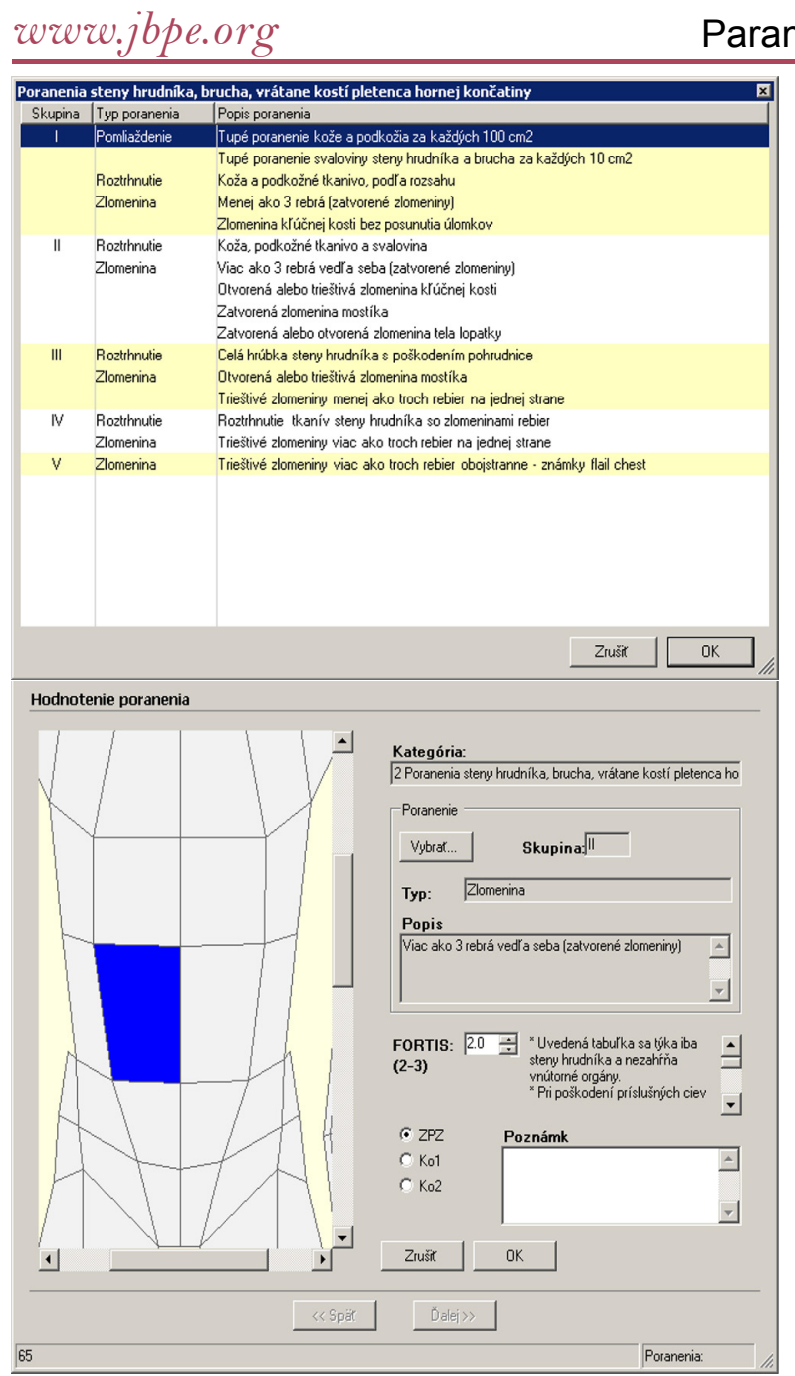

Figure 6: Example of classification tables and their use in the PC Fortis program

sification - is compatible with a multi-body system used for calculations in PC Crash, enabling an approximate analysis of forces acting during a collision on individual parts, or to recognize the consequences of the force acting upon a patient's body.

Assessment and Advantages of FORTIS system and PC FORTIS Program

FORTIS system represents a universal instrument usable in practice, which enables to substitute, for the needs of experts - analysts of traffic accidents, the medical verbal description of injuries with the description of injuries expressed in point scoring by means of their
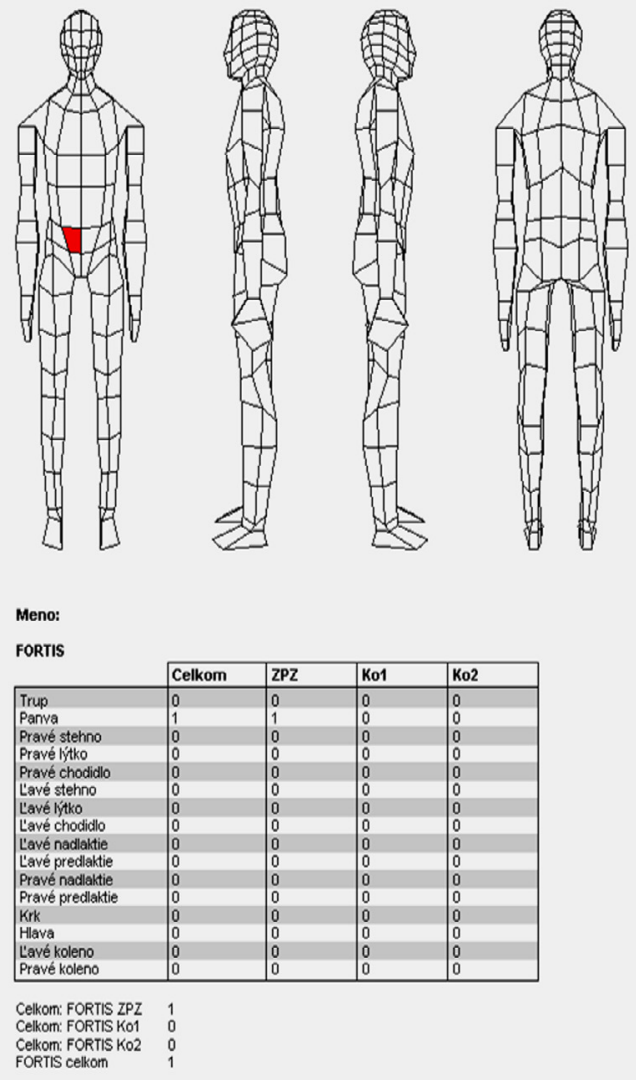

Figure 7: Illustration of FORTIS output after classification of injuries and localization of contacts

parametrization with high evidence value.

PC FORTIS program can be considered as a tool for expert practise at using the modified system FORTIS, and even in combination with video-recording from simulation of contacts of the pedestrian's body during collision and other outputs with their physical parameters from the PC Crash program or Virtual Crash program. The localisation and parametrization of injuries made in the PC FORTIS program by a medical examiner provides an option of direct visualisation of injuries as significant information for a traffic accident technical analyst. As its advantage can be considered, also the fact that it enables not only visualisation of injuries localisation, but also generation of individual signature of injuries (ratio of forces acting at the time of injury on individual parts of the body according to its distribution cor- 
responding to a calculation multi-body system in PC Crash) for a particular type of collision and for specific conditions, such as characteristics of pedestrian's body, his position, movement, type of vehicle, its speed, dynamics at the time of collision, etc. When conducting the research, the following data on energy distribution on the pedestrian's body at the time of collisions were also collected and calculated from the PC Crash program. We could state that for a particular collision such data have always individual features, from which it is possible to generate characteristic signatures.

A collision between a pedestrian and a vehicle represents a matrix of physical violence having an effect on the pedestrian's body and a matrix of the pedestrian's body's response to this violence. The stated matrices of impact of forces and other physical effects and of the pedestrian's body's responses are mutually dependent and are individual, identifiable and comparable for the specific collision.

It is not possible that in two different pedestrian/vehicle collisions the same forces have an effect on a pedestrian's body. Thus, the pedestrian's body's response (injuries) is always different.

In order to be able to recognize differences, or individual features, of a collision we may use a calculation simulation program - PC Crash, which not only calculates the course of a collision on a validated pedestrian model, but also generates the values of the physical violence having an effect on the pedestrian's body.

In order to recognize the individual features of the pedestrian's body's response (injuries), we may use the FORTIS system, which serves to perform the forensic universal parametrisation of pedestrian injuries by evaluating their injuries and which, based on forensic examiners' knowledge using a forensic scale, determines the effects affecting the surface of individual parts of the pedestrian's body in a special point scale.

In order to display the values, a so-called injury signature is used, which in connection with the localisation of the contacts on the pedestrian's body, allows for a comparison of these data with the values of the affecting physical violence generated from the calculation by means of PC Crash program as well as a comparison of the contact localisation in three-dimensional display (video recording) from the calculation.

The signature represents a visual expression of something - in this case a proportion of the values characterizing:

A. the physical effects on the surface of the pedestrian's body during a collision with a vehicle (signature of contacts)

B. the response of the pedestrian's body to these physical effects on the surface of his/her body determined on the basis of the forensic evaluation of the injuries observed (signature of injuries)

Then, the term signature of injuries means a visual expression of the localisation and extent of injuries on a pedestrian's body by means of FORTIS system, allowing a comparison with the signatures created from the outputs of a collision simulation and the evaluation of their compliance.

Individuality of Contacts during a Collision between a Pedestrian and a Vehicle

Within the performed research, the results obtained by NS of a backpropagation type indicate that the distribution of injuries on a pedestrian's body is always functionally dependent on the type of a collision, during which the pedestrian suffered them and that their signature (in this case of the energy distribution) be usable to identify a collision type. We may state that the experiment results obtained from this sample confirm the basic hypothesis, i.e. a possibility to recognize individual features of each individual collision between a pedestrian and a vehicle depending on the localization and type of pedestrian's injuries to a technically relevant extent [8]. 
A demonstration of a possibility to recognize the individual features of the contacts between a pedestrian and a vehicle during a collision and their comparisons with a signature of observed injuries parametrized in the FORTIS system in BF (Body Fortis)

orensic signature for sim 30-30R collision

Sim 30-30 - the impact speed of $30 \mathrm{~km} / \mathrm{hour}$, a direction of the pedestrian's movement - 30 degrees against the vehicle, R- pedestrian's stepped-out right leg, speed of the pedestrian - 4,6 km/hour (for all calculations). Figure 8

Sim 30-30R collision calculation - a display of pedestrian's contacts with a vehicle - Figure 9

Sim 30-30R collision calculation - a display of the calculated intensity of contacts - Figure 10

A signature of the contact forces for a sim 30-30R collision according to the calculation - Figure 11

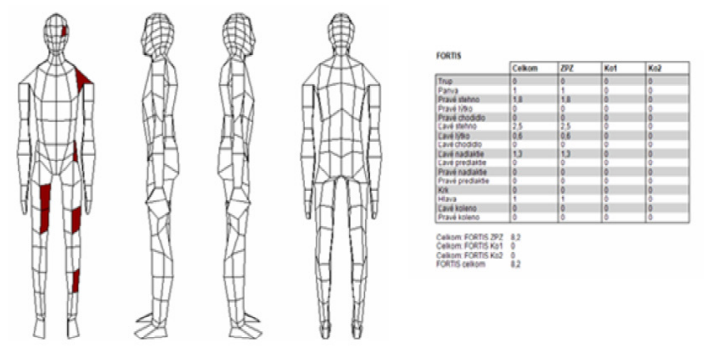

Pedestrian injury signature after a collision according to FORTIS (Fortis points) for sim 30-30

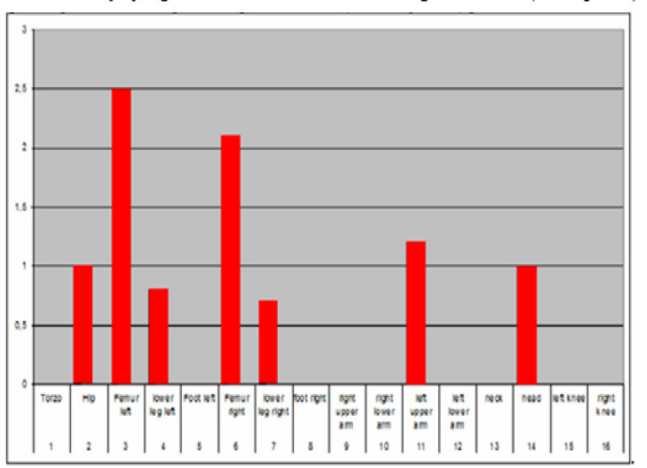

Figure 8: A display of the localization output, FORTIS system scoring and injury signature created based on the scoring of a basic injury to health (ZPZ)
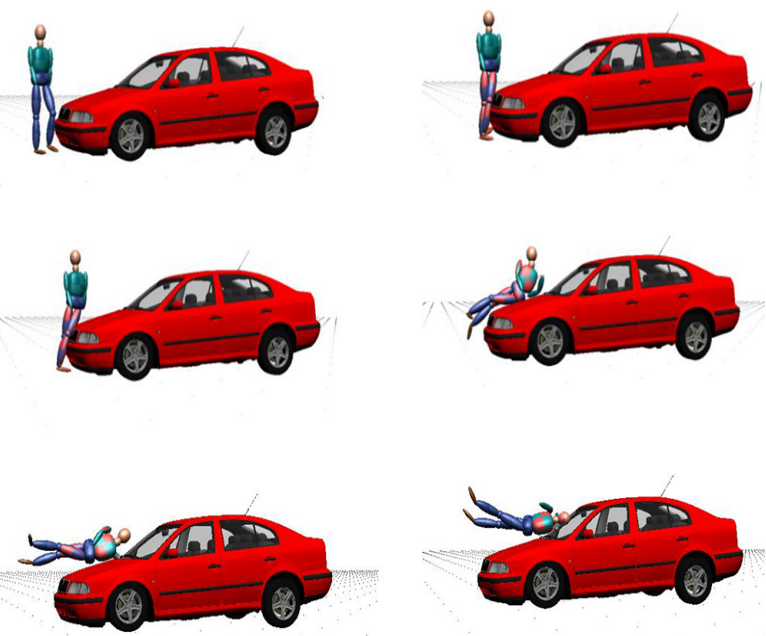

Figure 9: A display of the contacts of a collision between a pedestrian and a vehicle according to the sim 30-30R calculation

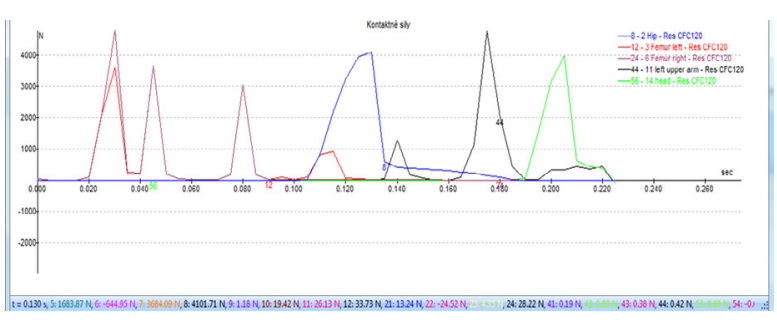

Figure 10: A display of the calculated intensity of contacts for pre sim 30-30R

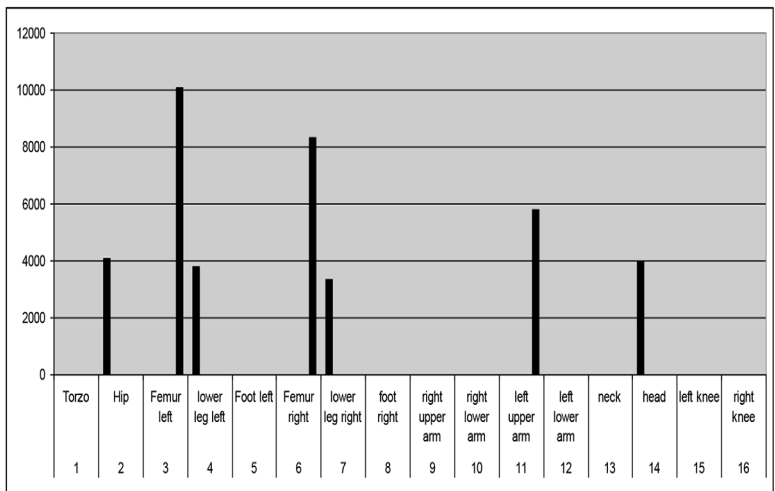

Figure 11: A display of contact signature sim 30-30 
A comparison of contact force signature and injury parameters for sim 30-30R collision Figure 12

For other comparable collisions, the following contact signatures were achieved:

A comparison of collision courses and contact force signatures for sim 30-30R, sim 3090R, sim 40-30R, sim 30-30L collisions - Figure 13.

\section{Discussion}

Based on the results of the research and its application in practice as they were presented in the article, it can be stated that the presented procedure represents a new perspective method of forensic assessment of injuries by means of a universal special dimensionless scale of the new FORTIS classification system, where the point values determine, based on the characteristics and the extent of the individual injuries, the physical violence that affected the contact area of the injured person's body at the time of the injury. The stated parameterization of the injuries as well as the localization of contacts using current advanced simulation programs designed to investigate traffic accidents are able to identify this injurious physical violence and, with the proven individuality of distribution and intensity of contacts, create, using the theory of injuries and contacts, the new possibilities to investigate the occurrence and course of traffic accidents.

\section{Conclusion}

It is apparent that for the needs of traffic accidents analysts while solving a vehicle-pe-
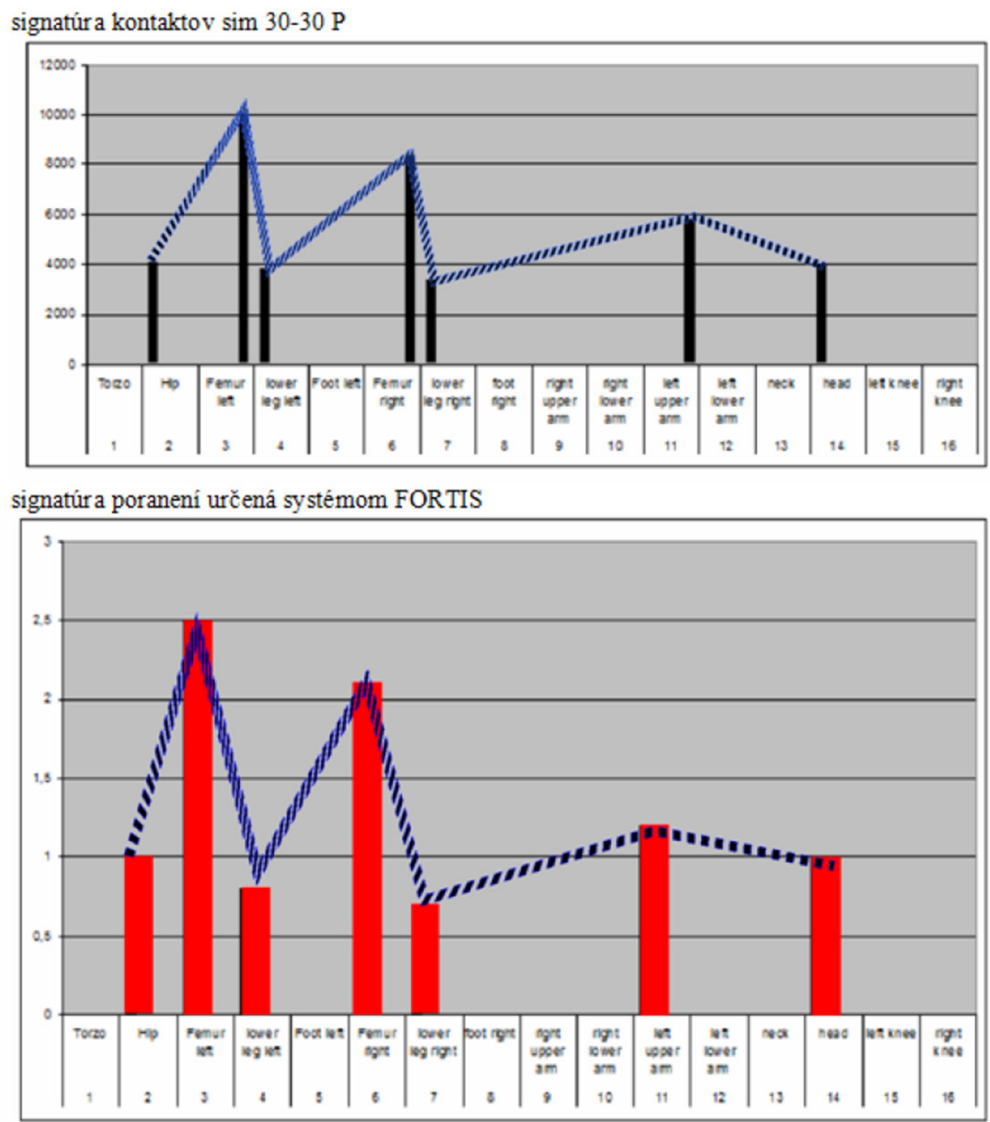

Figure 12: A display of a comparison of injury signature and contact signature for sim 30-30R 

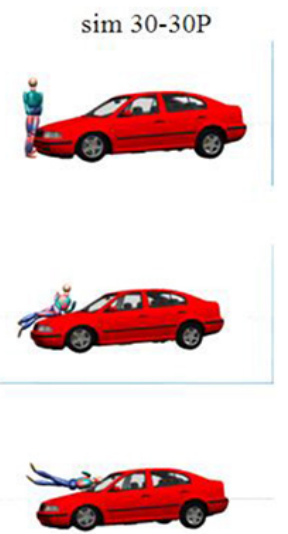

$\operatorname{sim} 30-30 \mathrm{P}$

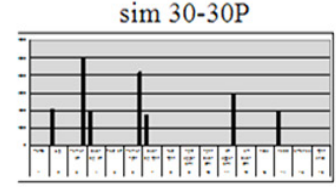

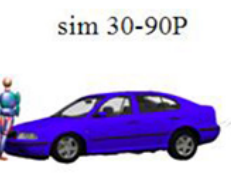
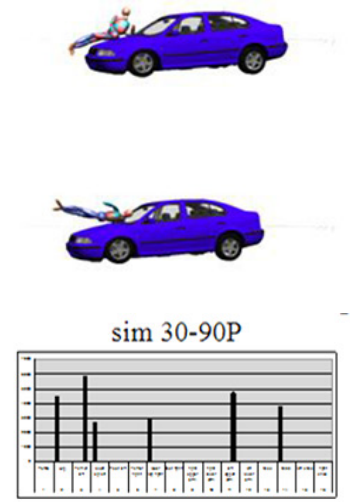

$\operatorname{sim} 40-30 \mathrm{P}$
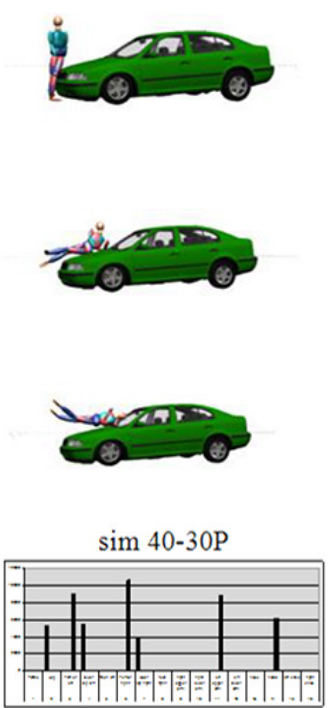

$\operatorname{sim} 30-30 \mathrm{~L}$
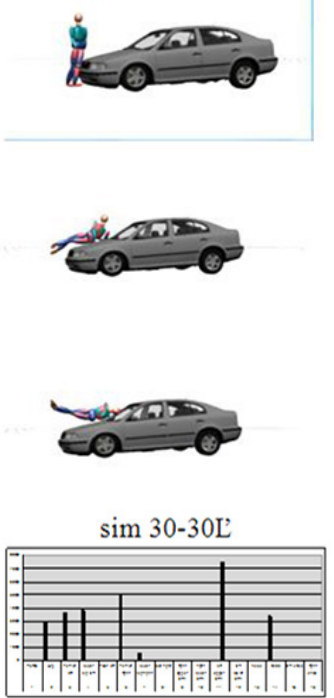

Figure 13: A display of the collision course and contact signatures for comparable collisions

destrian collision, an opportunity to compare a level of a compliance of the localisation of injuries at own calculations is created by the described means and procedures, and even a comparison of the compliance of distribution of the total energy and other physical influences acting upon collision on a pedestrian's body, using the presented way. The mentioned program has more options for being used in forensic medicine, as it covers not only a field of evaluation of traffic injuries but also all kinds of injuries and accidents (rail accidents, air accidents, violent crimes, etc.). In order to get more from the possibilities of its utilisation, it would be advisable to use FORTIS program at the first contact of a physician with a patient who has suffered an injury, and until the patient is fully recovered. This procedure would provide a complete documentation of traces on the body of an injured person as well as the assessment of other relevant facts. This procedure, based on the FORTIS system, the developed Theory of injury and contact signatures, has been used in the police practice in the Slovak Republic for several years and we may say that thanks to its utilisation, the evi- dencing process has been improved considerably, even in the cases in which it would not have been possible to achieve a clear conclusion by means of regular procedures. This can be attributed to the fact that the utilisation of FORTIS method makes it possible to evaluate the traces on a pedestrian's body in a way that it can be used for further evidencing by forensic engineers. The extent of a compliance of injury and contact signatures is strictly demonstrable, which is also accepted in court proceedings in proving and assessing the course of an accident in the legal system of Slovak Republic.

\section{Acknowledgment}

The authors of the publication are not in a mutual conflict of interests, and they agree with its publication. The manuscript is not in a conflict of interests with any natural or legal entity.

The presented research was funded by the authors themselves without any other support.

\section{Conflict of Interest}

None 


\section{References}

1. Moser A, Steffan H, Kasanický G. The pedestrian model in PC-crash-The introduction of a multi body system and its validation. SAE transactions. 1999:794-802.

2. Bobrov N, Longauer F, Szabo M, Mandelík J, Mandelíková Z. Standardization of Injury Parameters at Pedestrians Traffic Accidents in Forensic Medicine. Proceedings to the 80th Anniversary of Pasteur Hospital Foundation; 2004. p. 62-66 (in Slovak).

3. Mandelík J. Pedestrian's injuri parametrisation and its usage in description of traffic accidents. University of Žilina: PhD. Thesis; 2006. (in Slovak)

4. Bobrov N, Mandelík J, Havaj P. The possibilities of medico-legal evaluation of injuries and its usage in the interdisciplinary traffic accident solving. VŠBM Košice. 2017;185:21-9, 225. (in Slovak)

5. Bobrov N, Ginelliová A, Mandelík J, Longauer F, Mátyás T. The evaluation of soft tissue injury ex- tent at polytrauma in pedestrian traffic accident cases. Folia Societatis Medicinae Legalis Slovacae. 2012;2(1):13-17. (in Slovak)

6. Bobrov N, Ginelliová A, Mandelík J. The injury quantification in traffic accident cases: the injury signature. Proceedings of the 4th Czech and Slovak Congress of Forensic Medicine with international participation; 2014. p. 41-45 (in Slovak)

7. Mandelík J, Bobrov N. Parametrization of Injuries by the FORTIS System and its Utilisation at Solving Traffic Accidents with Pedestrians by the Police. American Scientific Research Journal for Engineering, Technology, and Sciences (ASRJETS). 2017;36(1):294-305.

8. Mandelík J, Bundzel M. Application of neural network in order to recognise individuality of course of vehicle and pedestrian body contacts during accidents. International Journal of Crashworthiness. 2018:1-14. 